\title{
Effect of internal reflections on the radiation properties and input admittance of integrated lens antennas
}

\section{Citation for published version (APA):}

Vorst, van der, M. J. M., de Maagt, P. J. I., \& Herben, M. H. A. J. (1999). Effect of internal reflections on the radiation properties and input admittance of integrated lens antennas. IEEE Transactions on Microwave Theory and Techniques, 47(9/1), 1696-1704. https://doi.org/10.1109/22.788611

DOI:

10.1109/22.788611

Document status and date:

Published: 01/01/1999

\section{Document Version:}

Publisher's PDF, also known as Version of Record (includes final page, issue and volume numbers)

\section{Please check the document version of this publication:}

- A submitted manuscript is the version of the article upon submission and before peer-review. There can be important differences between the submitted version and the official published version of record. People interested in the research are advised to contact the author for the final version of the publication, or visit the $\mathrm{DOI}$ to the publisher's website.

- The final author version and the galley proof are versions of the publication after peer review.

- The final published version features the final layout of the paper including the volume, issue and page numbers.

Link to publication

\section{General rights}

Copyright and moral rights for the publications made accessible in the public portal are retained by the authors and/or other copyright owners and it is a condition of accessing publications that users recognise and abide by the legal requirements associated with these rights.

- Users may download and print one copy of any publication from the public portal for the purpose of private study or research.

- You may not further distribute the material or use it for any profit-making activity or commercial gain

- You may freely distribute the URL identifying the publication in the public portal.

If the publication is distributed under the terms of Article 25fa of the Dutch Copyright Act, indicated by the "Taverne" license above, please follow below link for the End User Agreement:

www.tue.nl/taverne

Take down policy

If you believe that this document breaches copyright please contact us at:

openaccess@tue.nl

providing details and we will investigate your claim. 


\title{
Effect of Internal Reflections on the Radiation Properties and Input Admittance of Integrated Lens Antennas
}

\author{
Maarten J. M. van der Vorst, Student Member, IEEE, Peter J. I. de Maagt, Member, IEEE, \\ and Matti H. A. J. Herben, Senior Member, IEEE
}

\begin{abstract}
This paper begins with the modeling of the reflected waves within integrated lens antennas, which consist of a dielectric lens on which a planar antenna is mounted. It is demonstrated that if the relative dielectric constant of the lens is small $\left(\varepsilon_{r} \leq 4\right)$, the single- and double-reflected waves are sufficient to analyze the effect of the internal reflections. For small angles around boresight, these unwanted reflected fields mainly affect the cross-polar far-field pattern, while for large observation angles, both the co-polar and cross-polar patterns are significantly disturbed. It appears that by neglecting the internally reflected field contributions, the beam efficiency may be overestimated more than $10 \%$. In this paper, two types of matching layers are analyzed in order to reduce these unwanted reflections. It is demonstrated that the radiation performances of the integrated lens antennas with optimum-thickness and quarter-wavelength matching layer are almost equal. Even for low dielectric-constant lenses, the beam efficiency can be increased by over $10 \%$. Finally, it is demonstrated that the internal reflections may also have a strong effect on the antenna admittance, which can only be reduced partly by the use of a matching layer.
\end{abstract}

Index Terms - Integrated lens antennas, internal reflections, matching layers.

\section{INTRODUCTION}

A NEW generation of instruments, at millimeter- and submillimeter-wavelengths, included in both Earth observation and scientific missions, is under consideration. In the current European Space Agency (ESA) Earth observation studies, millimeter and submillimeter limb-sounding instruments (e.g., MASTER and SOPRANO) are already foreseen to use frequencies up to $1 \mathrm{THz}$. Also projected in this frequency band are astronomy missions (e.g., FIRST) in which the frequency range may even extend to above $3 \mathrm{THz}$.

The specifications of these instruments are very stringent; crucial points being very high beam efficiency, high Gaussian beam efficiency (Gaussicity), and very low receiver noise temperature. These lead to the need for low-loss antennas with very high-quality antenna patterns and low sidelobes.

Manuscript received September 22, 1998. This work was supported in part by the European Space Agency under Contract 11653/95/NL/PB.

M. J. M. van der Vorst was with the Faculty of Electrical Engineering, Eindhoven University of Technology, Eindhoven, The Netherlands. He is now with the TNO Physics and Electronics Laboratory, The Hague, The Netherlands.

P. J. I. de Maagt is with the European Space Research and Technology Centre, European Space Agency, Noordwijk, The Netherlands.

M. H. A. J. Herben is with the Faculty of Electrical Engineering, Eindhoven University of Technology, Eindhoven, The Netherlands.

Publisher Item Identifier S 0018-9480(99)06609-0.
If the receiver is used for spacecraft applications, the requirement of robustness naturally arises. Furthermore, certain atmospheric constituents are somewhat polarized and this requires knowledge of the polarization purity of the antenna patterns. Traditionally, waveguide-based front-ends are used in the millimeter-wave regions, where semiconductor-insulator-semiconductor (SIS) or a Schottky diode and usually a horn (corrugated, diagonal, or Potter) acts as standard mixing element and feed, respectively. In this conventional technology, the feed, mixer, demultiplexing circuitry, and local oscillator are all realized as separate devices. As the frequency increases, it becomes more and more complex to manufacture these devices and to assemble them, due to their small sizes.

However, the small size can be turned into an advantage if the dimensions and tolerances required become compatible to those achieved by lithography. In this case, a planar structure, which integrates the antenna, mixer, local oscillator, and all peripheral circuitry onto one single substrate, turns into an alternative solution that could be competitive with the conventional technology. Furthermore, these integrated frontends promise advantages such as reproducibility, robustness, possible extension into an array antenna, and low production cost if they are produced in large quantities. A problem that prevented a previous widespread use of this technology in millimeter-wave and submillimeter-wave receivers is their perceived poor performance. However, with an increasing understanding of planar designs, this is gradually changing, and it is now recognized that competitive performance can be achieved. One of the problems that has been encountered is the fact that planar antennas on dielectric substrates couple power into substrate modes, and since these do not contribute to the primary radiation pattern, substrate-mode power is generally considered as a loss mechanism. The problem of substrate modes can be overcome by making the substrate either very thin or infinitely thick. If the planar antenna, e.g., a slot antenna, is mounted on a substrate that is much thinner than the free-space wavelength, almost half of its input power will be radiated in the backward direction. An infinitely thick substrate can be simulated by using some kind of lens shape such as the extended hemisphere (e.g., hyperhemisphere or synthesized ellipse) or a true ellipse. In case of an elliptical lens, most of the radiation goes through the dielectric into the forward direction. One of the advantages of such a lens is that the beamwidth of the planar radiator is reduced, which makes it 


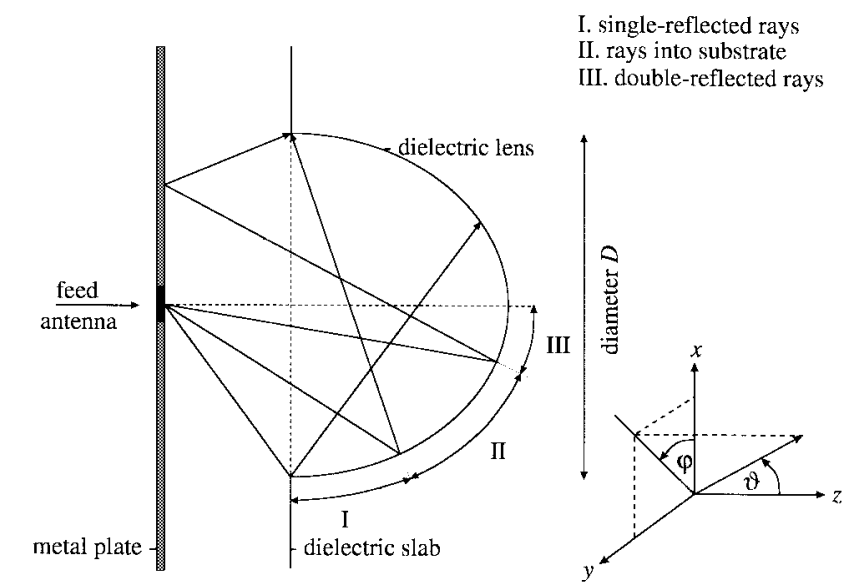

Fig. 1. Antenna geometry and angular domain of three types of reflected rays.

easier to use these antennas (with lens) in practical systems. A number of people have already successfully used the dielectriclens concept for millimeter- and submillimeter-wave receivers [1]-[3].

A disadvantage of all lens antenna designs is that they suffer from reflection "losses," which may strongly affect the far-field radiation properties and the input admittance of the integrated lens antenna. Therefore, this paper starts with the modeling of these reflected waves, followed by an analysis of their effect on the co-polar and cross-polar far-field antenna patterns. Subsequently, two types of matching layers are investigated to reduce the unwanted internal reflections. The performance of the lens antennas with these matching layers are compared on the basis of co-polar and cross-polar patterns, beam efficiency, and Gaussian beam efficiency, which are generally considered to be the prime antenna properties for the previously mentioned applications. The paper is concluded with an analysis of the effect of internal reflections on the input admittance of integrated lens antennas.

\section{INTERNAL REFLECTIONS}

\section{A. Geometry and Types of Rays}

The general geometry of an integrated lens antenna is depicted by its two-dimensional cross section, shown in Fig. 1. As can be seen from this figure, the antenna consists of a planar radiating element, dielectric slab, and focusing lens, whose center is chosen to lie on top of the slab. It is assumed that the lens has the same dielectric constant as the slab, and that its shape is either elliptical or hemispherical. In order to calculate the far-field radiation patterns (co-polar and cross-polar) of this antenna, a geometrical optics (GO) approach is applied inside the lens and physical optics (PO) outside the lens [4].

In Silver [5], the Fresnel field and far field at observation point $P$ is described in terms of the PO integral as

$$
\begin{aligned}
\underline{E}(P)=\frac{-j}{4 \pi \omega \varepsilon_{0}} \iint_{S} & {\left[\left(\underline{J}_{s} \cdot \nabla\right) \nabla+k_{0}^{2} \underline{J}_{s}\right.} \\
& \left.\quad-j \omega \varepsilon_{0}\left(\underline{M}_{s} \times \nabla\right)\right] \frac{e^{-j k_{0} r}}{r} d S
\end{aligned}
$$

and

$$
\begin{aligned}
\underline{H}(P)=\frac{-j}{4 \pi \omega \mu_{0}} \iint_{S}[ & \left(\underline{M}_{s} \cdot \nabla\right) \nabla+k_{0}^{2} \underline{M}_{s} \\
& \left.\quad+j \omega \mu_{0}\left(\underline{J}_{s} \times \nabla\right)\right] \frac{e^{-j k_{0} r}}{r} d S
\end{aligned}
$$

where $S$ is the lens surface and $r$ the distance between $P$ and a point on $S$. The equivalent electric and magnetic current densities are denoted by $\underline{J}_{s}$ and $\underline{M}_{s}$, respectively. To justify the use of GO inside the lens, the diameter of the lens should be large compared to the wavelength in the dielectric. This also justifies the assumption that all rays originate from the center of the feed antenna. A common problem arising in these lens antennas is that the incident wave is partly reflected at the lens-air interface. Of course, the corresponding reflected power should not really be treated as loss, but as interference, because part of the reflected wave can leave the lens after one or more internal reflections and, therefore, affect the radiation pattern.

Examination of Fig. 1 reveals three different angular domains inside the lens of which domains I and III actually contribute to the field outside the dielectric lens. The angles determining the different domains are computed using a zerofinding algorithm, where the actual function, representing the derivative of the ray-path length, depends on the shape of the lens and slab thickness. The first group of internal reflected rays, the so-called single-reflected rays (angular domain I), have been reflected once at the surface of the lens before they contribute to the far-field radiation pattern. For rays having an angle of propagation within angular domain II, it can be seen that, after reflection at the lens surface, they propagate directly into the substrate and it is there where they will most likely be trapped as substrate modes. If the infinitely extended substrate is replaced by a finite substrate under the lens, the extra reflected fields can be included in a similar way as the single- and double-reflected fields. However, the influence of this extra surface will not be significant because the incident power from the feed is low and the reflection coefficients are small (smaller angle of incidence). Finally, angular domain III consists of rays that hit the lens for a second time after complete reflection at the metal plate. It is worth noting that this metal plate is present if the feed is either a (double) slot or a (double) dipole with backing reflector [6], but it is not a limitation of this model. The double dipole with backing reflector consists of a two single dipole elements on a dielectric lens with a metallic reflector behind them. In between the dipoles and metal plane, the same dielectric is used as for the lens. Without a metal plate, there are still double-reflected rays, although their contribution will be smaller.

Both above-mentioned internally reflected field contributions can be treated as follows. Via a ray-tracing technique inside the lens, the induced equivalent magnetic and electric current densities just outside the lens can be found. In order to account for the divergence losses of the reflected waves a (divergence) factor is included, which describes the amplitude variation along a reflected ray [7]. Once the separate equivalent current density contributions $\underline{J}_{s}$ and $\underline{M}_{s}$ just outside the lens 
TABLE I

Power Distribution (in Percentages) of an Elliptical Lens Illuminated By a Double-Dipole Feed with BACKing Reflector

\begin{tabular}{c||c|c|c|c|c}
\hline$\varepsilon_{r}$ & $P_{\text {trans }}$ & $P_{\text {refl }}$ & $P_{I}$ & $P_{I I I}$ & $P_{I I}$ \\
\hline \hline 2.31 & 76.9 & 23.1 & 22.8 & 0.2 & 0.1 \\
\hline 4.00 & 76.6 & 23.4 & 20.8 & 0.8 & 1.8 \\
\hline 9.80 & 67.8 & 32.2 & 13.4 & 7.9 & 10.9 \\
\hline 11.7 & 65.1 & 34.9 & 11.8 & 11.3 & 11.8 \\
\hline \hline$\varepsilon_{r}$ & $P_{I}^{t}$ & $P_{I}^{r}$ & $P_{I I I}^{t}$ & $P_{I I I}^{r}$ & $P_{n a f}$ \\
\hline \hline 2.31 & 22.0 & 0.8 & 0.1 & 0.1 & 0.9 \\
\hline 4.00 & 14.7 & 6.1 & 0.1 & 0.7 & 6.8 \\
\hline 9.80 & 4.2 & 9.2 & 0.8 & 7.1 & 16.3 \\
\hline 11.7 & 3.1 & 8.7 & 0.9 & 10.4 & 19.1 \\
\hline
\end{tabular}

are determined, (1) and (2) are applied to calculate each farfield contribution, decomposed into its co-polar and cross-polar components. The co-polar and cross-polar components are according to Ludwig's third definition [8].

It is noted that the dielectric losses are assumed to be zero in this paper, but of course, they can be included very easily into the ray-tracing procedure. The loss tangent of the dielectric materials studied is in the order of $1-5 \times 10^{-4}$ [9], resulting in dielectric losses of less than $1 \%$ for the direct incident rays and less than $2 \%$ for the internally reflected rays. Therefore, by taking the dielectric losses into account, the results will not change noticeably.

\section{B. Numerical Results}

By including the previously described internal reflected field contributions, the impact of the reflected waves on the antenna performance can now be determined. As an example, a planar double dipole with a backing reflector will be used in combination with an elliptical lens. The dielectric material is selected to be high-density polyethylene (HDP), quartz, alumina, or silicon with an $\varepsilon_{r}$ of 2.31, 4.0, 9.8, and 11.7, respectively. The dimensions of the planar feed are $0.5 \lambda_{d}$ for the length and $0.4 \lambda_{d}$ for the distance between the elements. The metallic backing reflector is placed at a distance of $0.25 \lambda_{d}$ behind the array. This configuration results in good rotationally symmetric feed patterns into the dielectric. To determine the significance and completeness of the singleand double-reflected fields for different dielectric materials, Table I shows the relative powers of the different types of reflected waves. The diameter of the lens is not relevant here, as the power values only depend on the lens shape and, thus, on the dielectric lens material. All power values are given in percentages of the total power incident on the lens.

From Table I, it can be seen that the reflection "losses" $\left(P_{\text {reff }}\right)$ increase only slightly when HDP is replaced by quartz, thus increasing the dielectric constant. The reason for this is that, although the feed patterns in the dielectric are equal, the lens illuminations are quite different for the two antenna designs due to the different lens shapes. By selecting a higher dielectric-constant material as quartz, the feed location shifts toward the center of the elliptical lens, which results in smaller angles of incidence and, thus, lower reflection coefficients at the lens surface. Furthermore, the distribution of the reflected power shows that this power is mainly contained within

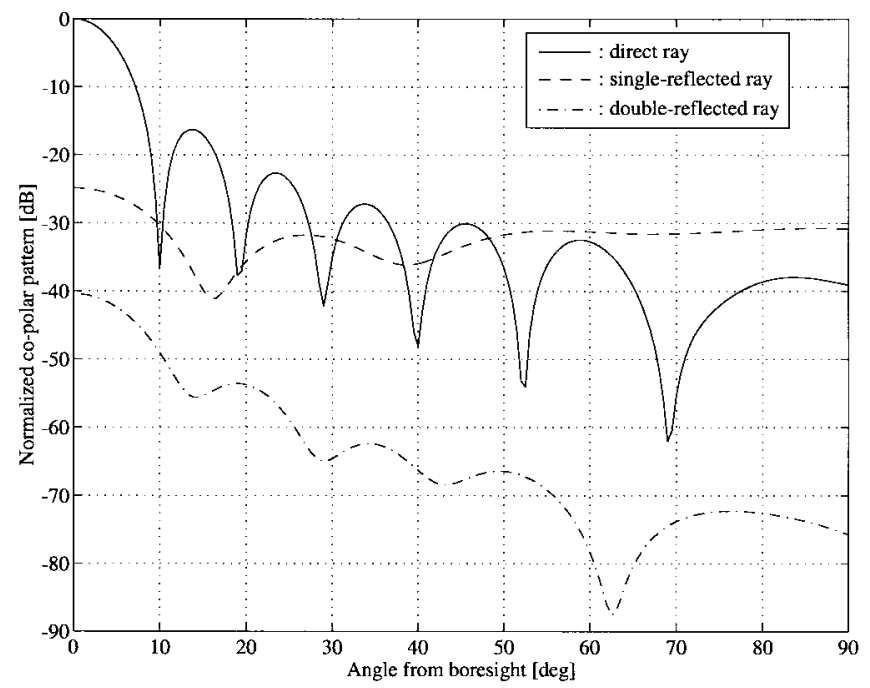

Fig. 2. Normalized co-polar patterns, in the diagonal plane, of a 4.0-mm-diameter elliptical HDP lens antenna illuminated by a double-dipole antenna with backing reflector at $500 \mathrm{GHz}$.

the single-reflected fields $\left(P_{\mathrm{I}}\right)$, whereas the double-reflected field contribution is negligible for both materials. Another observation that can be made is that, for these two antenna designs, just a small amount of power goes directly into the substrate $\left(P_{\mathrm{II}}\right)$. These findings together with the higher dielectric constant of quartz compared with HDP result in less internal reflected power $\left(P_{\mathrm{I}}^{t}+P_{\mathrm{III}}^{t}\right)$, which is contributing to the far-field radiation pattern for quartz. The amount of the internal reflected power that is reflected for a second time at the lens surface $\left(P_{\mathrm{I}}^{r}+P_{\mathrm{III}}^{r}\right)$ is denoted by $P_{\text {naf }}$ and stands for the power that is not accounted for in the present model. In fact, this reflected power belongs to the higher order internal reflected fields and, in Table I, it is shown that for the higher dielectric constant material, more power is not taken into account. This means that the accuracy of the radiation patterns obtained with the inclusion of the single-reflected and doublereflected fields is higher for HDP than for quartz (e.g., for quartz $P_{\text {naf }}$ is $6.8 \%$ ).

Additional computations for hyperhemispherical lenses, made of various materials, demonstrate that they suffer from less reflection "losses" than do elliptical lenses and that, for these special extended hemispherical lenses as well, a larger part of the reflected power is propagating into the substrate. This proves once again that in addition to the relative dielectric constant, the shape of the lens is an important parameter in relation to the influence of the internally reflected waves on the vectorial far-field pattern.

As an example, the co-polar and cross-polar patterns of a 4.0-mm-diameter elliptical HDP lens antenna, which is illuminated by the double-dipole antenna with backing reflector, are calculated in the diagonal plane $\left(\varphi=45^{\circ}\right)$ at $500 \mathrm{GHz}$. The three separate normalized pattern contributions are depicted in Figs. 2 and 3, which reveal that the single-reflected ray contribution is the most important contribution, as can also be observed from the power distribution given in Table I. Nevertheless, for the co-polar radiation, the influence of the internal reflections is expected only to appear for larger angles 


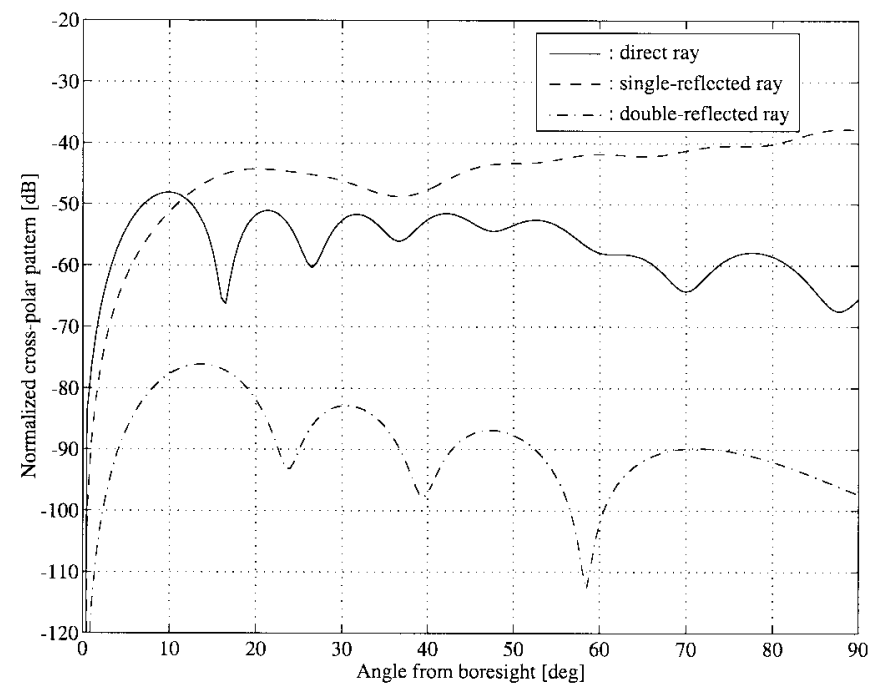

Fig. 3. Normalized cross-polar pattern, in the diagonal plane, of a 4.0-mm-diameter elliptical HDP lens antenna illuminated by a double-dipole antenna with backing reflector at $500 \mathrm{GHz}$.

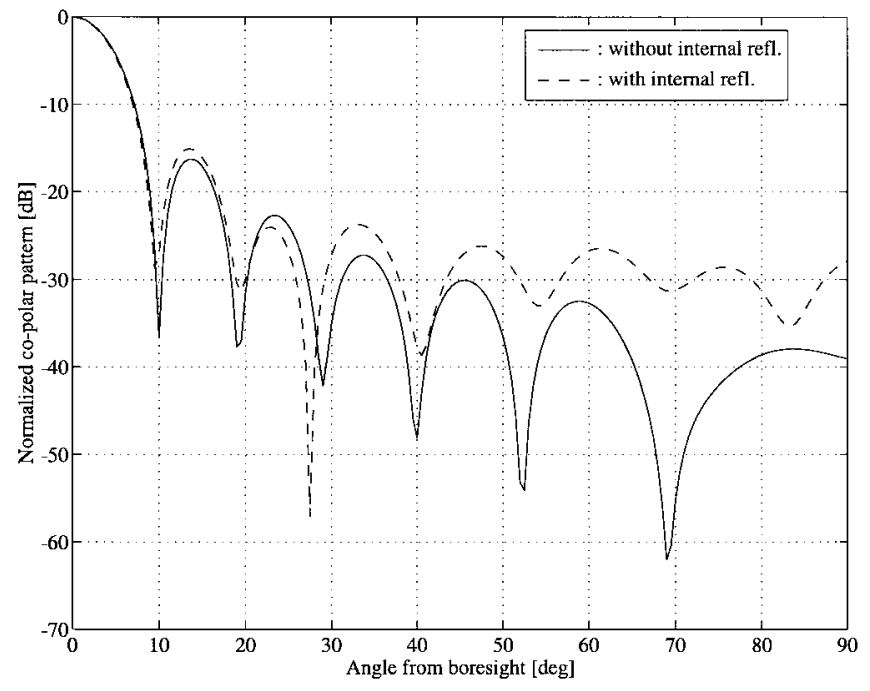

Fig. 4. Normalized overall co-polar patterns, in the diagonal plane, of a 4.0-mm-diameter elliptical HDP lens antenna illuminated by a double-dipole antenna with backing reflector at $500 \mathrm{GHz}$.

from boresight. To get a better idea of the lens effect, the overall radiation patterns are depicted in Figs. 4 and 5, where the three contributions are added with their appropriate phases.

The overall far-field radiation patterns show that, for small angles from boresight, mainly the cross-polar pattern is affected by the internal reflection contributions. However, by looking at the patterns for larger far-field angles, one observes that both the co-polar and cross-polar radiation patterns change significantly. The increase of the antenna sidelobes will result in an overestimation of the beam efficiency if the internally reflected field contributions are not taken into account. Table I shows that, for HDP, the overestimate is greater than $10 \%$. The degradation of the radiation properties of integrated lens antennas due to the mismatch at the lens-air interface can be reduced by placing a matching layer at the interface. Therefore, Section III deals with the design and performance of matching layers for integrated lens antennas.

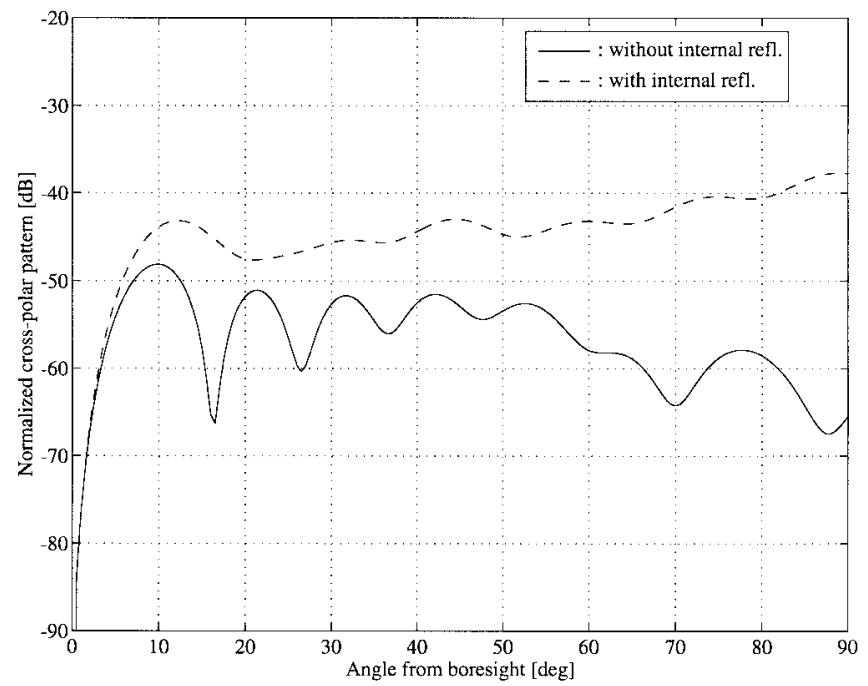

Fig. 5. Normalized overall cross-polar pattern, in the diagonal plane, of a 4.0-mm-diameter elliptical HDP lens antenna illuminated by a double-dipole antenna with backing reflector at $500 \mathrm{GHz}$.

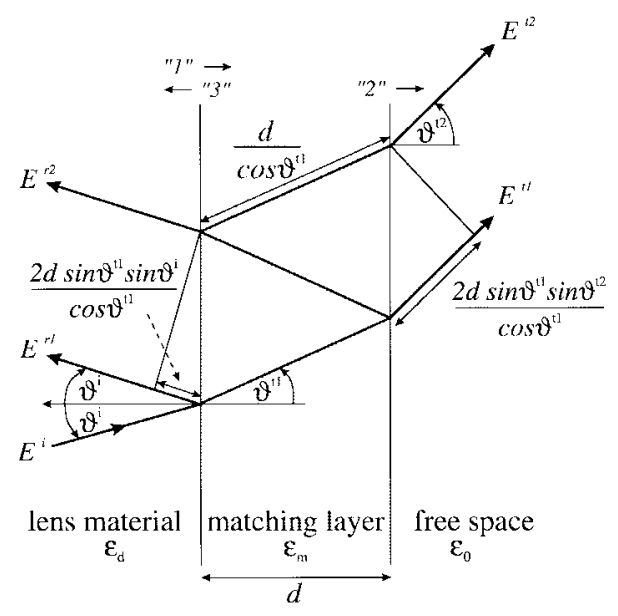

Fig. 6. Ray tracing of a plane wave through a lossless dielectric matching layer.

\section{MATCHING LAYER}

\section{A. Design and Modeling}

For the analysis of the matching layer, two assumptions are made. First, both the incident wave and shape of the matching layer are assumed to be locally plane. Second, the matching layer is theoretically modeled using an infinite number of internal reflections. In order to comply with the first assumption, it should be possible to approximate the "infinite" reflection and transmission coefficients by a finite series of internal reflections, and it was found that this can be done to sufficient accuracy by taking into account four to five different ray contributions.

In this section, the following notation is adopted (see Fig. 6): subscript "1" refers to the boundary from the lens to the matching layer, subscript "2" denotes the boundary from the matching layer to free space, and finally, subscript " 3 " is used for the boundary from matching layer back to the lens material. 
For the derivation of the "infinite" reflection and transmission coefficients, the configuration of Fig. 6 and the dielectric layer model described in [10] are used. This leads to the following equation for the "infinite" reflection coefficient:

$$
\begin{aligned}
R_{\infty} & =R_{1}+T_{1} T_{3} R_{2} P_{d}^{2} P_{a} \sum_{m=0}^{\infty}\left(R_{2} R_{3} P_{d}^{2} P_{a}\right)^{m} \\
& =R_{1}+\frac{T_{1} T_{3} R_{2} P_{d}^{2} P_{a}}{1+R_{1} R_{2} P_{d}^{2} P_{a}}
\end{aligned}
$$

while for the "infinite" transmission coefficient, it is possible to write

$$
T_{\infty}=T_{1} T_{2} P_{d} \sum_{m=0}^{\infty}\left(R_{2} R_{3} P_{d}^{2} P_{a}\right)^{m}=\frac{T_{1} T_{2} P_{d}}{1+R_{1} R_{2} P_{d}^{2} P_{a}}
$$

where the relation $R_{3}=-R_{1}$ is substituted. In (3) and (4), two phase terms are introduced, of which $P_{d}$ describes the phase shift that results from the propagation of a ray through the matching layer. The second phase shift $P_{a}$ describes the phase shift that is due to the path length difference between subsequent rays in a far-field observation point, which is caused by the fact that the rays leave the slab at different points. The corresponding path lengths are depicted in Fig. 6 .

Equations (3) and (4) can be used for both perpendicular and parallel polarization if the appropriate Fresnel reflection $\left(R_{i}\right)$ and transmission $\left(T_{i}\right)$ coefficients are substituted. These Fresnel coefficients are functions of the refractive indexes and the angle of incidence.

It is well known that the choice of a quarter-wavelength matching layer $\left(d=\lambda_{m} / 4\right.$ and $\left.\varepsilon_{m}=\sqrt{\varepsilon_{d} \varepsilon_{0}}\right)$ together with a normal incident field at the lens to matching layer boundary, will result in a zero reflected field. However, in the integrated lens antenna design, shown in Fig. 1, the incident wave has an angle of incidence that is changing over the lens surface. Therefore, for this application, the quarter-wavelength layer does not reduce the reflections to a minimum. An optimumthickness matching layer for this case will be designed in the following section.

It should be noted that the phase of the "infinite" transmission coefficient changes over the lens surface because the angle of incidence changes and, therefore, the matching layer can affect the focusing property of the elliptical lens.

\section{B. Optimum-Thickness Layer}

For the specific application of a matching layer in combination with integrated lens antennas, an optimum design can be found that reduces the reflected power at the lens-air interface to a minimum. In this section, it is assumed that the matching layer is made of a homogeneous material with a fixed dielectric constant. Without this constraint, the reflection losses could, in principle, be reduced to zero, but such a layer is very difficult (or impossible) to make. In defining the optimumthickness matching layer for a specific lens antenna design, it is important to determine the thickness as a function of the angle of incidence, at which the "infinite" transmission coefficient is maximal. By substitution of $P_{d}^{2} P_{a}=\exp (j \xi)$ into (4) and by taking the derivative of $\left|T_{\infty}\right|$ with respect to $\xi$, the maximum and minimum values of the "infinite" transmission coefficient can be found. First, the absolute value of the complex variable $T_{\infty}$ is calculated as

$$
\left|T_{\infty}\right|=\frac{T_{1} T_{2}}{\sqrt{1+\left(R_{1} R_{2}\right)^{2}+2 R_{1} R_{2} \cos \xi}}
$$

where the Fresnel transmission coefficients $T_{1}$ and $T_{2}$ both have real values because only lossless dielectric materials are considered and the lens design is such that the angle of incidence never exceeds the critical angle. For the derivative of $T_{\infty}$, the following equation is obtained:

$$
\frac{d\left|T_{\infty}\right|}{d \xi}=\frac{R_{1} R_{2} T_{1} T_{2} \sin \xi}{\left(1+\left(R_{1} R_{2}\right)^{2}+2 R_{1} R_{2} \cos \xi\right)^{3 / 2}} .
$$

Next, the maxima of $T_{\infty}$ can be found by taking into account that $T_{1}$ and $T_{2}$ are both positive numbers and that the product $R_{1} R_{2}$ is always positive in the case of perpendicular polarization and positive in the case of parallel polarization if the angle of incidence is smaller than the Brewster angle $\left(R_{2, \text { par }}=0\right)$. With this knowledge and (6), the maxima of the "infinite" transmission coefficient appear for $\xi= \pm \pi$. Only those solutions that correspond to the thinnest matching layer structure are considered here.

The following step is to calculate the optimum thickness $d_{\text {opt }}$ of the matching layer as a function of the angle of incidence. Equating the combination of the phase factors $P_{d}^{2} P_{a}$ and the previous result obtained for $\xi$ yields

$$
\frac{-2 j k_{m} d_{\mathrm{opt}}+2 j k_{0} d_{\mathrm{opt}} \sin \vartheta^{t 1} \sin \vartheta^{t 2}}{\cos \vartheta^{t 1}}= \pm j \pi
$$

where $k_{m}$ denotes the wavenumber in the matching layer. Taking into account that the left-hand side of (7) is negative, the final result for the thickness profile of the matching layer is found as

$$
\frac{d_{\mathrm{opt}}}{\lambda_{m}}=\frac{1}{4 \cos \vartheta^{t 1}}=\frac{1}{4 \sqrt{1-n_{d} \sin ^{2} \vartheta^{i}}} .
$$

This equation is applicable for both perpendicular and parallel polarization. Furthermore, it can be proven that, for this optimum-thickness matching layer design, the "infinite" reflection and transmission coefficients for both polarizations are equal, and they are depicted in Fig. 7.

For angles of incidence larger than the Brewster angle (at which $R_{2, \text { par }}=0$ ), the optimum-thickness matching layer no longer has the highest transmission coefficient for parallel polarization. This can be seen in Fig. 7, where it appears that the no-matching layer design (for parallel polarization) results in the lowest reflection coefficient.

\section{Effect on Beam Pattern}

In this section, the influence of the matching layer, either an optimum-thickness (opt.) or a quarter-wavelength (q.w.) layer, on the co-polar and cross-polar radiation patterns and some other important antenna properties will be determined. In the examples, three different cases will be compared with each other. Initially, no matching layer is used and, as a consequence, the internal reflections have to be included. In the second case, a matching layer is applied, which has a constant thickness of a quarter wavelength across the surface of the lens. However, if one is interested in a matching layer with very low 


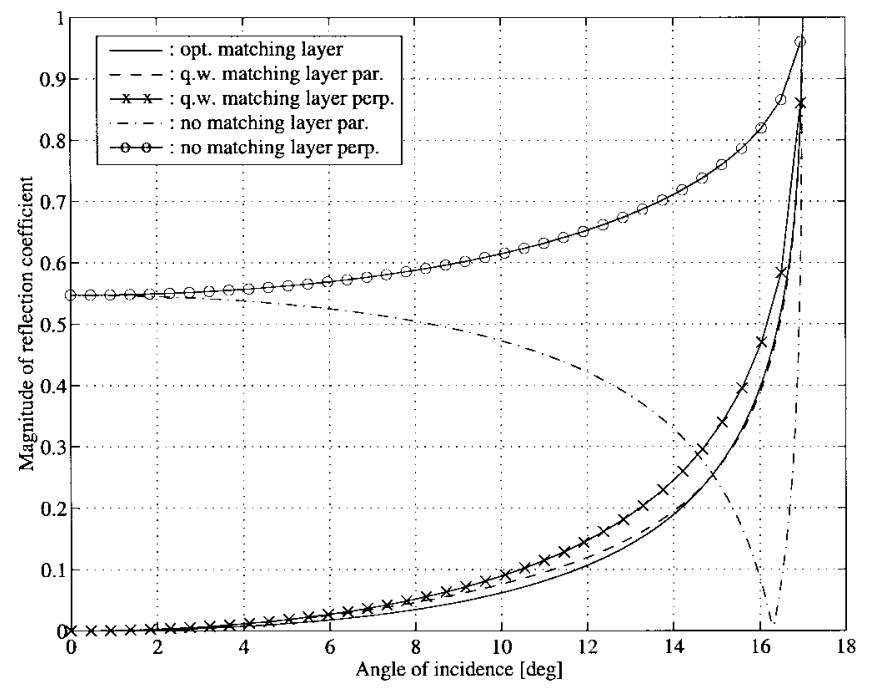

Fig. 7. Magnitude of reflection coefficients for a silicon substrate.

reflection "losses" at the lens surface, then the matching layer should be shaped according to the optimum-thickness profile of (8), and the third case treats this matching layer type. Two planar feeds will be used, both with either a silicon $\left(\varepsilon_{r}=11.7\right)$ or quartz $\left(\varepsilon_{r}=4.0\right)$ elliptical lens. The lens diameter and the frequency are chosen as $4.0 \mathrm{~mm}$ and $500 \mathrm{GHz}$, respectively. In the first example, a silicon lens is used, which is illuminated by a double-dipole feed with backing reflector. As in Section II-B, the dimensions of this feed antenna design are $l=0.5 \lambda_{d}$ and $\Delta d=0.4 \lambda_{d}$, where $l$ is the length of the antenna and $\Delta d$ is the distance between the elements. Again, the backing reflector is placed a quarter-wavelength behind the feed. The co-polar and cross-polar radiation pattern are calculated and depicted in Figs. 8 and 9, respectively.

As a second example, a silicon dielectric lens is used, which is illuminated by a double-slot feed antenna. For this planar feed, $l=0.35 \lambda_{0}$ and $\Delta d=0.185 \lambda_{0}$ result in a good rotationally symmetric pattern into the dielectric. Figs. 10 and 11 show the co-polar and cross-polar radiation pattern of this antenna.

Comparing the radiation patterns of the integrated lens antennas with and without a matching layer reveals that both the directivity and sidelobes are increased when a quarterwavelength or an optimum-thickness layer is applied. Furthermore, the shape of the main lobe and first few sidelobes (co-polar pattern) does not seem to change significantly, but the cross-polar patterns within this angle region are strongly affected by the matching layers. It is also clear from these figures that the radiation patterns of the antenna with an optimum matching layer design almost coincide with those of an antenna with a quarter-wavelength matching layer.

In case of the double-dipole feed with backing reflector, the cross-polar level increases when a matching layer is used, while for the double-slot feed, it decreases. The explanation for this is the polarization dependency of the reflection coefficients for the different matching layer designs. For every design, the polarization of the incident field at the lens is modified differently (Fig. 7). In the case of the double-dipole feed with backing reflector, a matching layer on top of the lens can only

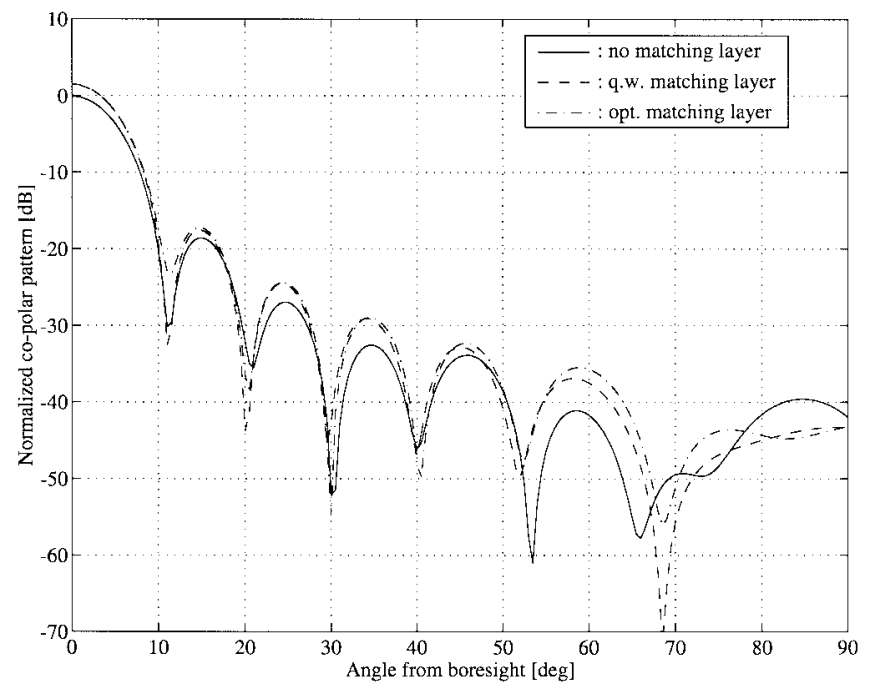

Fig. 8. Normalized co-polar patterns in the diagonal plane of a 4.0-mm-diameter elliptical silicon lens antenna, illuminated by a double-dipole feed with backing reflector at $500 \mathrm{GHz}$.

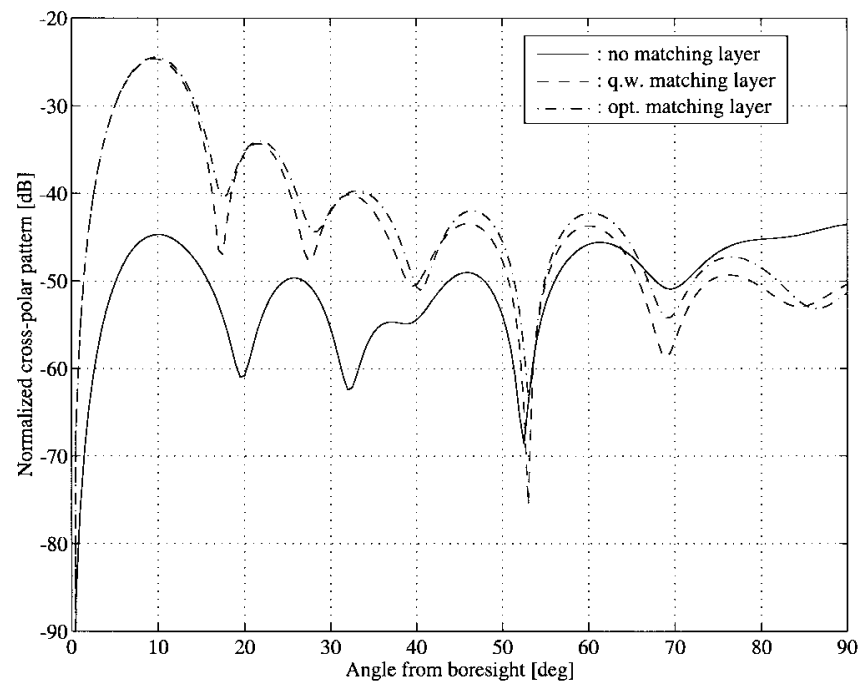

Fig. 9. Normalized cross-polar patterns in the diagonal plane of a 4.0-mm-diameter elliptical silicon lens antenna, illuminated by a double-dipole feed with backing reflector at $500 \mathrm{GHz}$.

worsen the cross-polar performance because the polarization efficiency is close to unity for the uncoated lens. On the other hand, for the double-slot feed, the application of a matching layer increases the cross-polar performance. In Table II, the polarization efficiencies are given for the different designs, and from that table, the same conclusions can be drawn as from Figs. 8 to 11 .

Next, some other important antenna parameters are also determined and shown in Table II for both a silicon and a quartz dielectric lens antenna. For the double-slot antenna on the quartz lens, the distance between the radiating elements is increased to $0.31 \lambda_{0}$ in order to realize a good rotationally symmetric pattern into the dielectric. If the double-slot feed on quartz is replaced by a planar double-dipole feed, the dimensions $l=0.5 \lambda_{d}$ and $\Delta d=0.4 \lambda_{d}$ still hold.

It is clear from the table that the quartz lens has the lowest spillover efficiency $\left(\eta_{s}\right)$ and Gaussian beam efficiency 


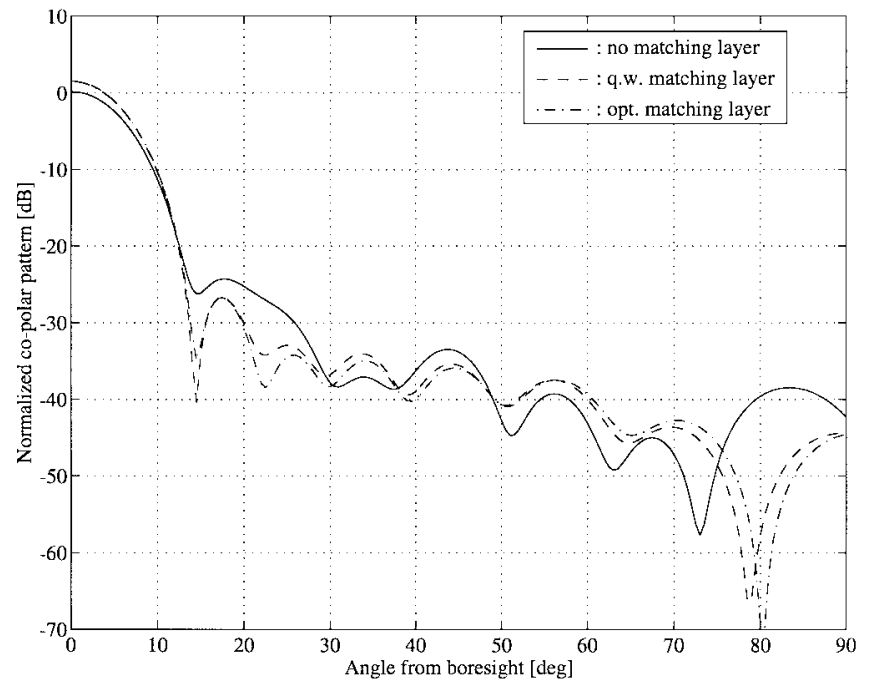

Fig. 10. Normalized co-polar patterns in the diagonal plane of a 4.0-mm-diameter elliptical silicon lens antenna, illuminated by a double-slot feed at $500 \mathrm{GHz}$.

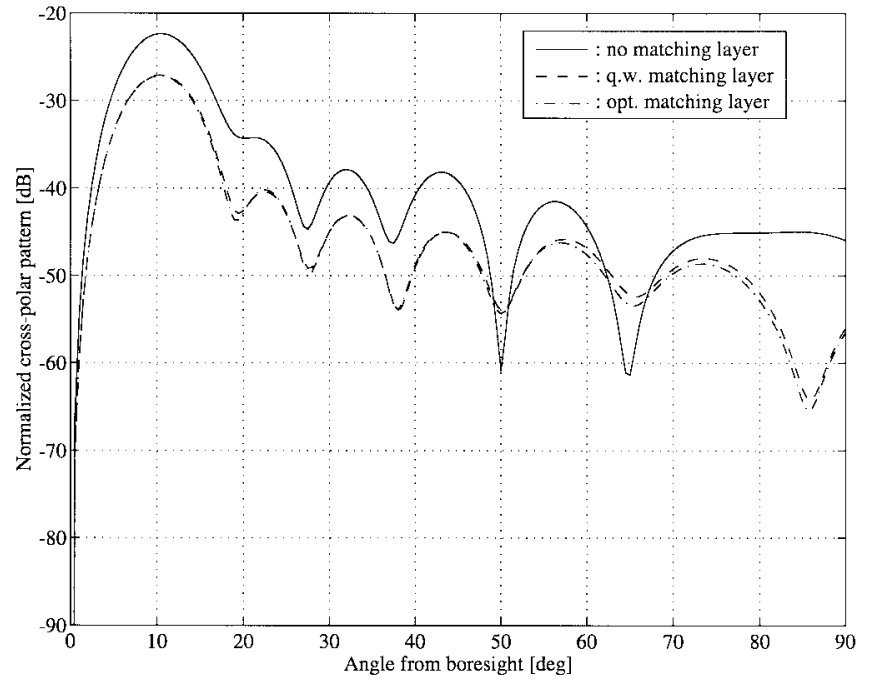

Fig. 11. Normalized cross-polar patterns in the diagonal plane of a 4.0-mm-diameter elliptical silicon lens antenna, illuminated by a double-slot feed at $500 \mathrm{GHz}$.

$\left(\eta_{\text {gauss }}\right)$. For a definition of the antenna efficiencies used, see the Appendix. Furthermore, Table II reveals that the matching layer increases the beam efficiency $\left(\eta_{\text {beam }}\right)$ by more than $5 \%$ for the silicon lens and by even more than $10 \%$ for the quartz one. The largest improvement is obtained by using a quarter-wavelength matching layer.

\section{INPUT ADMITTANCE}

\section{A. Modeling}

In the previous sections, the influence of the internal reflected waves on the radiation pattern was the key issue. However, as the feed is located at or close to the focus of the lens (ellipsoid), the input admittance can also be affected by the internal reflections. The rays from angular region I will converge to a single point (caustic) after their second reflection inside a focused elliptical lens. Thus, these waves can be considered as the main source of the influence on the input admittance of the planar feed. In this section, some results will be given concerning the admittance change due to the lens surface.

For convenience, in the following, only the double-slot feed will be considered, and for the modeling of the input admittance [11] is used. If the lens effect is included in the modeling, an extra admittance is needed due to the reflections within the lens antenna [12]. For this admittance, the following induced EMF method formula, equivalent to the one derived in [13], can be given:

$$
Y_{\text {lens }}=-\frac{1}{V_{0}} \int_{-w / 2}^{w / 2} \int_{-l / 2}^{l / 2} \underline{M}(x, y) \cdot \underline{H}^{r}(x, y) d x d y
$$

with $V_{0}$ the voltage at the antenna terminals. The $y$-integration can be simplified because the reflected magnetic field will be nearly constant over the width of the slot feed $(w / l \ll 1)$. In (9), each single-slot element is assumed to be located in the focus, but, in fact, it is placed slightly off-axis $( \pm \Delta d / 2)$. Thus, for the computation of the admittance due to the lens, the incident (reflected) magnetic field and the current distribution along the slots are required. Using the method of moments (MoM), the magnetic current distribution $\left(M_{x}\right)$ along the slots can be found, where only an $x$-component is assumed due to the small width of the slots. Since the slots are positioned close to a caustic of the rays that are reflected twice at the lens surface, GO cannot be used to predict the magnetic field and, therefore, (2) needs to be applied. It is noted that the equivalent current densities are now determined from the reflected fields and not the transmitted fields. Also, $\varepsilon_{0}$ and $k_{0}$ need to be replaced by $\varepsilon_{0} \varepsilon_{r}$ and $k_{0} \sqrt{\varepsilon_{r}}$, respectively.

If the matching layer is included in the integrated lens antenna design, the same theoretical model can be applied, but then the Fresnel reflection coefficients have to be replaced by the "infinite" reflection coefficients from (3). The effect of the matching layer will be that the magnitude of the reflected magnetic field decreases and also an extra phase is introduced for each ray preventing the existence of a caustic near the slots.

\section{B. Numerical Results}

Like the results for the radiation patterns, the effect of the internal reflections on the input admittance can be computed for the case with and without a matching layer. To come to an admittance value, the slots need to have a certain width and a value of $5 \%(w / l=0.05)$ of the length is assumed. To clearly demonstrate the effect of the lens, the off-resonance double slot on a quartz lens is used as an example. This double-slot design is the same as used in Section III-C. In Figs. 12 and 13, the results are showed for the real and imaginary parts of the slot input admittance in the case of an infinite dielectric [11], a lens without a matching layer, and a lens with a quarter-wavelength matching layer.

The first observation that can be made is that the influence of the lens on the admittance of the double slot is significant for this particular design. Secondly, it appears that the application of a quarter-wavelength matching layer does not completely 
TABLE II

Efficiencies and Relative First Sidelobe Levels (D-Plane) of a 4.0-MM Elliptical Lens Antenna at 500 GHz

\begin{tabular}{c||c|c|c|c|c|c|c|c}
\hline $\begin{array}{c}\text { planar feed } \\
\text { type }\end{array}$ & $\begin{array}{c}\text { matching layer } \\
\text { type }\end{array}$ & $\varepsilon_{T}$ & $\begin{array}{c}\text { first sidelobe } \\
\text { level }(\mathrm{dB})\end{array}$ & $\begin{array}{c}\eta_{s} \\
(\%)\end{array}$ & $\begin{array}{c}\eta_{t r} \\
(\%)\end{array}$ & $\begin{array}{c}\eta_{p} \\
(\%)\end{array}$ & $\begin{array}{c}\eta_{\text {beam }} \\
(\%)\end{array}$ & $\begin{array}{c}\eta_{\text {gauss }} \\
(\%)\end{array}$ \\
\hline \hline double dipole & no & 11.7 & -18.5 & 99.0 & 65.2 & 99.9 & 83.5 & 88.0 \\
\hline double dipole & constant & 11.7 & -19.1 & 99.0 & 93.1 & 99.4 & 88.9 & 88.2 \\
\hline double dipole & optimum & 11.7 & -18.6 & 99.0 & 94.2 & 99.2 & 89.0 & 88.4 \\
\hline double dipole & no & 4.00 & -16.9 & 93.5 & 76.6 & 99.8 & 68.2 & 82.0 \\
\hline double dipole & constant & 4.00 & -17.0 & 93.5 & 88.3 & 99.4 & 82.1 & 84.1 \\
\hline double dipole & optimum & 4.00 & -14.7 & 93.5 & 90.6 & 98.9 & 78.2 & 84.9 \\
\hline double slot & no & 11.7 & -24.8 & 83.9 & 70.4 & 99.1 & 89.7 & 91.1 \\
\hline double slot & constant & 11.7 & -28.5 & 83.9 & 97.2 & 99.7 & 96.1 & 94.1 \\
\hline double slot & optimum & 11.7 & -28.4 & 83.9 & 97.6 & 99.7 & 95.8 & 94.2 \\
\hline double slot & no & 4.00 & -19.1 & 68.3 & 79.4 & 99.0 & 75.5 & 84.7 \\
\hline double slot & constant & 4.00 & -18.9 & 68.3 & 91.3 & 99.4 & 87.5 & 86.6 \\
\hline double slot & optimum & 4.00 & -16.4 & 68.3 & 93.1 & 99.4 & 84.4 & 87.1 \\
\hline
\end{tabular}

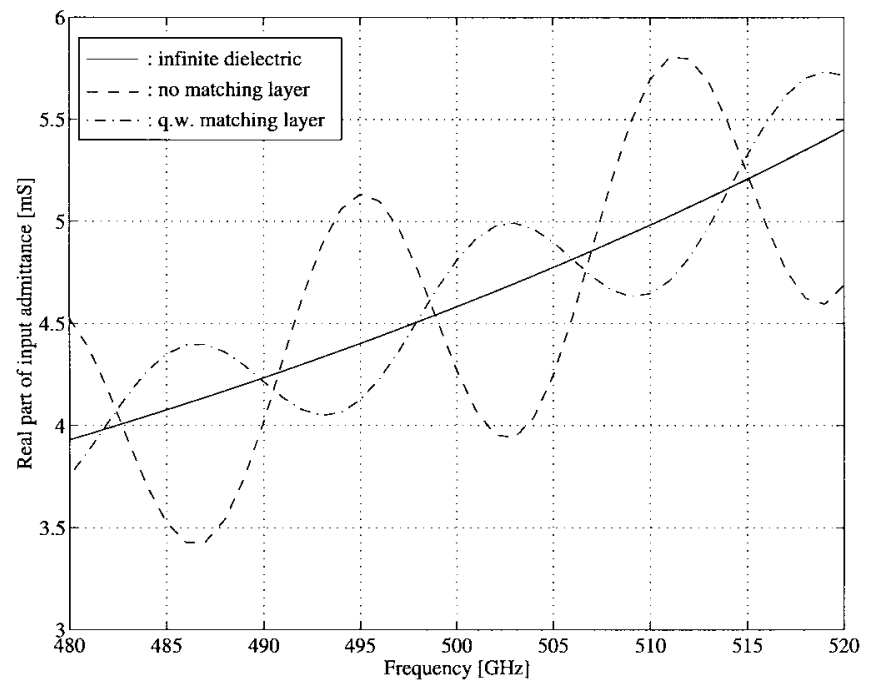

Fig. 12. Real part of input admittance of a double slot on a 4.0-mm-diameter elliptical quartz lens antenna.

remove the lens effect. This can be explained by examining Fig. 7, where it is shown that, for large angles of incidence, the "infinite" reflection coefficient is not really smaller than the Fresnel reflection coefficients. This fact, together with the knowledge that the rays corresponding to the single-reflected fields have large angles of incidence, leads to the observed reduction in the lens effect. Additional computer simulations made clear that the main reason for the reduced lens effect, in case a matching layer is used, is the extra phase of the "infinite" reflection coefficient. Due to this extra phase, the focus is no longer a caustic for the rays that are reflected twice at the lens surface and, consequently, the reflected magnetic field is weaker.

Computations for the silicon lens antenna with double-slot feed showed an even smaller reduction of the lens effect when a quarter-wavelength matching layer was put on top of the lens.

\section{CONCLUSIONS}

Comparing the shape of the radiation patterns of the integrated lens antennas with and without the inclusion of internal reflections reveals that in the case of elliptical lenses without a matching layer, the differences found in the main

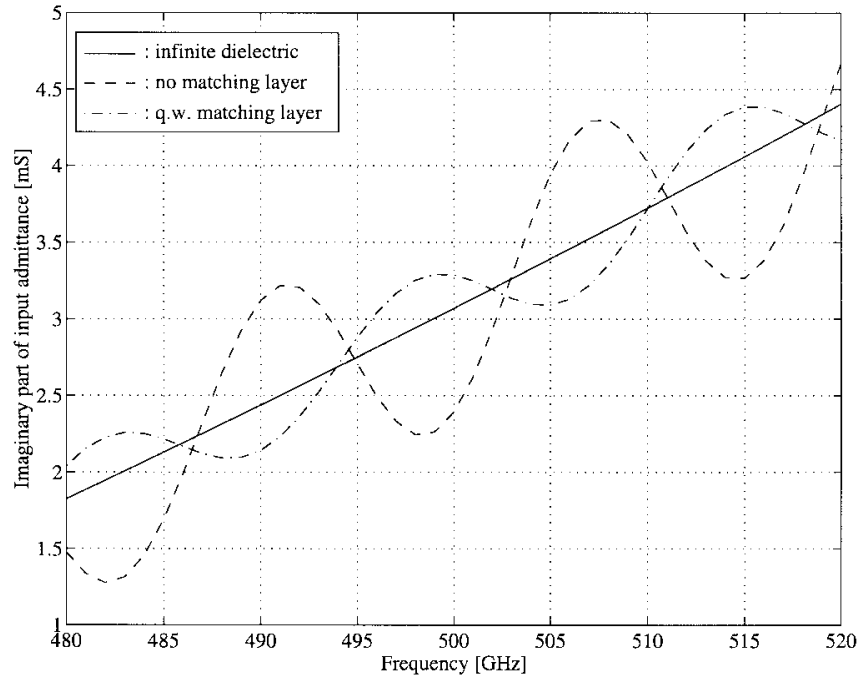

Fig. 13. Imaginary part of input admittance of a double slot on a 4.0-mm-diameter elliptical quartz lens antenna.

lobe and the first few sidelobes of the co-polar patterns are negligible. However, for larger angles from boresight, the influence of the internal reflections on the co-polar patterns becomes significant. This results in an incorrect estimation of the beam efficiency if the internal reflections are not taken into account. Furthermore, it has become clear that the internal reflected rays can also have a major effect on the shape and level of the cross-polar radiation patterns. It was found that for the integrated lens antennas with $\varepsilon_{r} \leq 4$, the single- and double-reflected rays are sufficient to describe the influence of the reflected fields on the antenna performance.

To reduce the internal reflected power to approximately $5 \%$, a matching layer is needed at the lens-air interface. In this paper, an expression was derived for the thickness profile of the optimum matching layer design for integrated lens antennas. It appears, however, that for the high dielectric-constant lens materials $\left(\varepsilon_{r} \geq 4\right)$, the beam efficiency can be improved best by applying a quarter-wavelength matching layer. Even for low dielectric-constant lenses, the beam efficiency can be increased by over $10 \%$.

Another conclusion that can be drawn from this paper is that the co-polar pattern does not change significantly for the main lobe and the first few sidelobes, but the cross-polar pattern is 
strongly affected by the matching layer. Whether the matching layer has a positive or negative effect on the cross-polar pattern strongly depends on the antenna configuration.

Finally, from the input admittance computations, it can be concluded that the effect of the lens can be significant, e.g., $10 \%$ for the double-slot design, if the input admittance of the planar feed on a dielectric half-space is not too large. The application of a quarter-wavelength layer only reduces the lens effect by a factor of about two because of the large angles of incidence of the rays from region I at the second reflection point.

\section{APPENDIX}

For a measure of the performance of (aperture) lens antennas, different efficiencies are used. The efficiencies appearing in this paper are: 1) spillover efficiency $\left(\eta_{s}\right)$, which is the power incident on the lens divided by the total power radiated by the planar feed; 2$)$ transmission efficiency $\left(\eta_{\mathrm{tr}}\right)$, which is the power transmitted through the lens divided by the total power incident on the lens; 3) polarization efficiency $\left(\eta_{p}\right)$, which is the power in the co-polar pattern divided by the total power radiated by the lens; 4) (main) beam efficiency $\left(\eta_{\text {beam }}\right)$, which is the power in the main beam divided by the total power radiated by the lens; and 5) Gaussian beam efficiency $\left(\eta_{\text {gauss }}\right)$, which is the coupling of the lens antenna to a first-order Gaussian beam. A note on the Gaussian beam efficiency is that, in this paper, the efficiency is calculated from the far-field pattern and the representation of the Gaussian beam is slightly different from [4]. Filipovic uses a paraxial approximation for the Gaussian far field, but here, the Gaussian far-field pattern is found by transforming the Gaussian field pattern at the beam waist position [14] using PO. This results in the following expression:

$$
\begin{aligned}
\underline{E}(r, \vartheta, \varphi)=\frac{j k A w_{0}^{2}}{2 r} \exp (-j k r) \exp \left(\frac{-\sin ^{2} \vartheta}{\vartheta_{0}^{2}}\right) \\
\cdot \exp \left(\frac{j \pi \vartheta^{2}}{\vartheta_{1}^{2}}\right) \underline{e}_{c o}
\end{aligned}
$$

where $\underline{e}_{C O}$ is $\hat{e}_{\vartheta} \sin \varphi+\hat{e}_{\varphi} \cos \vartheta \cos \varphi$ for a double slot parallel to the $x$-axis and $\hat{e}_{\vartheta} \cos \varphi-\hat{e}_{\varphi} \cos \vartheta \sin \varphi$ for a double dipole with backing reflector along the same axis.

\section{ACKNOWLEDGMENT}

The authors thank A. Neto for useful discussions on the topic of the input admittance.

\section{REFERENCES}

[1] J. Zmuidzinas and H. G. LeDuc, "Quasi-optical slot antenna SIS mixers," IEEE Trans. Microwave Theory Tech., vol. 40, pp. 1797-1804, Sept. 1992.

[2] R. M. Heeres et al., "First beam pattern measurements of integrated lens antennas at $500 \mathrm{GHz}$," in Proc. 2nd ESA Workshop Millimeter-Wave Technol. Applicat., 1998, pp. 327-332.

[3] S. S. Gearhart and G. M. Rebeiz, "A monolithic $250 \mathrm{GHz}$ Schottkydiode receiver," IEEE Trans. Microwave Theory Tech., vol. 42, pp. 2504-2511, Dec. 1994

[4] D. F. Filipovic, S. S. Gearhart, and G. M. Rebeiz, "Double-slot antennas on extended hemispherical and elliptical silicon dielectric lenses," IEEE Trans. Microwave Theory Tech., vol. 41, pp. 1738-1749, Oct. 1993.
[5] S. Silver, Microwave Antenna Theory and Design. New York: McGraw-Hill, 1949, ch. 3

[6] M. J. M. van der Vorst, "Design and analysis of integrated lens antennas," Faculty Elect. Eng., Eindhoven Univ. Technol., Eindhoven, The Netherlands, Graduation Rep., 1995.

[7] S. W. Lee et al., "Refraction at a curved dielectric interface: Geometrical Optics solution," IEEE Trans. Microwave Theory Tech., vol. MTT-30, pp. 12-19, Jan. 1982.

[8] A. C. Ludwig, "The definition of cross-polarization," IEEE Trans. Antennas Propagat., vol. AP-21, pp. 116-119, Jan. 1973.

[9] J. W. Lamb, "Miscellaneous data on materials for millimeter and submillimeter optics," Int. J. Infrared Millim. Waves, vol. 17, pp. 1997-2034, 1997.

[10] J. M. van Houten and M. H. A. J. Herben, "Analysis of a phasecorrecting Fresnel-zone plate antenna with dielectric/transparent zones," J. Electromag. Waves Applicat., vol. 8, pp. 847-858, 1994.

[11] G. V. Eleftheriades and G. M. Rebeiz, "Self and mutual admittance of slot antennas on a dielectric half-space," Int. J. Infrared Millim. Waves, vol. 14, pp. 1925-1946, 1993

[12] A. Neto, S. Maci, and P. J. I. de Maagt, "Reflections inside an elliptical dielectric lens antenna," Proc. Inst. Elect. Eng., vol. 145, pp. 243-247, 1998.

[13] C. A. Balanis, Antenna Theory: Analysis and Design. New York: Wiley, 1982, ch. 8

[14] P. F. Goldsmith, "Quasioptical techniques at millimeter and submillimeter-wavelengths," Infrared and Millimeter-Waves Vol. 6, K. J. Button, Ed. New York: Academic, 1982.

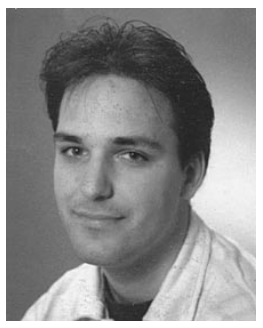

Maarten J. M. van der Vorst (S'95) was born in Oudenbosch, The Netherlands, on November 12, 1970. He received the M.Sc. and the Ph.D. degrees from Eindhoven University of Technology, Eindhoven, The Netherlands, in 1995 and 1999, respectively, both in electrical engineering.

$\mathrm{He}$ is currently with the TNO Physics and Electronics Laboratory, The Hague, The Netherlands. His research interests include millimeter-wave and submillimeter-wave integrated antennas, microwaves and radar technology.

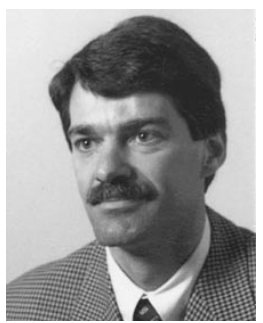

Peter J. I. de Maagt (S'88-M'88) was born in Pauluspolder, The Netherlands, in 1964. He received the M.Sc. and Ph.D. degrees from Eindhoven University of Technology, Eindhoven, The Netherlands, in 1988 and 1992, respectively, both in electrical engineering.

$\mathrm{He}$ is currently with the European Space Research and Technology Centre, European Space Agency, Noordwijk, The Netherlands. His research interests are in the area of millimeter- and submillimeterwave reflector and planar integrated antennas, quasioptics, photonic bandgap antennas, and millimeter-wave and submillimeterwave components.

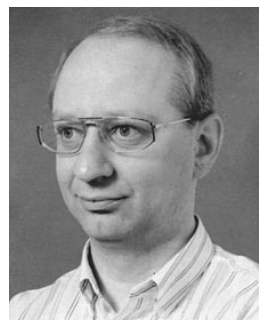

Matti H. A. J. Herben (S'80-M'83-SM'88) was born in Klundert, The Netherlands, in 1953. He received the M.Sc. degree (with distinction) in electrical engineering and the $\mathrm{Ph} . \mathrm{D}$. degree in technical sciences from Eindhoven University of Technology (EUT), Eindhoven, The Netherlands, in 1978 and 1984, respectively.

Since 1978, he has been with the Radiocommunications Group, EUT, where he is currently an Associate Professor. His research interests and publications are in the areas of design and numerical analysis of reflector and lens antenna systems, radio interference reduction, electromagnetic wave propagation on terrestrial and satellite links, remote sensing of the turbulent troposphere, and microwave radiometry.

Dr. Herben is a member of the Institute of Dutch Engineers (KIvI), The Netherlands Electronics and Radio Society (NERG), and the Dutch URSI Committee. 